\title{
WIRKLICHKEITSBRÜCHE. THEORIE UND ANALYSE MIT BLICK AUF TEXTE DER FRÜHEN MODERNE UND POSTMODERNE
}

\begin{abstract}
Crashes with reality - A narrative phenomenon in literary fiction of the early modern and the postmodern period

The article concentrates on the collision between plural concepts of reality in narrative fiction. For that purpose it focuses on theoretical aspects of world construction as well as on issues concerning the notions of character and narrative instances from a narratological point of view. Narrative collisions depend on heterogeneous, pluriregional or instable worlds, characters' deficits in knowledge and problematic perception, metalepses and the exposure and handling of the borderline between two or more concepts of reality. To illustrate these theoretical preliminaries the article attempts to show how texts functionalise such structures to create narrations of transition. Arnold Höllriegel's The Films by Princess Fantoche (1913) was written in the context of the early modern period. It correlates the collision of 'realities' to general questions of the modern crisis about whether to follow traditional ways or to find new ones. In Benjamin Stein's Replay (2012) subjectivity is raised as a problem insofar as the narrating character loses himself in entangled time-circles and delivers an unreliable narration. Stein discusses the postmodern game of intertextuality, technologism, eroticism, and self-reflection as well as the loss of the narrative and the wish for its renewal.
\end{abstract}

\section{Zur Theorie der literarischen Wirklichkeitsbrüche: Welten, Divergenzen, Kontexte}

Wirklichkeitsbrüche $(\mathrm{Wb})$ resultieren aus der Gegenüberstellung zweier (oder mehr) Wirklichkeitskonzepte im Rahmen eines fiktionalen Erzähltextes ${ }^{1}$, die unaufgelöst nebeneinander bestehen bleibt oder aber zugunsten eines der präsentierten Konzepte aufgelöst wird. Wb basieren auf der Konstitution der erzählten Welt, der narrativen Vermittlung und/oder der Figurenhandlung und korrelieren mit außertex-

\footnotetext{
${ }^{1}$ Zur theoretischen Fundierung pluraler Wirklichkeitskonzeptionen vgl. die großangelegte Studie Dominik Orths: Narrative Wirklichkeiten. Eine Typologie pluraler Realitäten in Literatur und Film, Marburg 2013, insb. 121-248; eine Übersicht seiner Typologie pluraler Wirklichkeiten findet sich auf S. 231.
} 
tuellen Diskursen; d.h. es handelt sich um Textphänomene, die Rückschlüsse auf das jeweilige Literatur-, Denk- und Wissenssystem einer Epoche zulassen.

Gebunden an die narrative Vermittlung, die mittels explizit und implizit Thematisiertem einen (thematischen) Vordergrund erzeugt (und einen nicht thematisierten Horizont des Dargestellten voraussetzt), lassen sich zunächst vier Möglichkeitsbereiche fiktionaler Wirklichkeitsbildung bestimmen: 1. Homogene Welten etablieren einheitliche Realitätssysteme, heterogene uneinheitliche. Einheitlich sind Systeme, sofern sie in Bezug auf die Variablen der Möglichkeit, der Wahrscheinlichkeit und der Notwendigkeit eine (und nur eine) Geltungsrichtung aufweisen. Uneinheitlich sind sie hingegen dann zu nennen, wenn sie mehrere, voneinander abweichende Geltungsrichtungen etablieren. 2. Während uniregionale Welten ein einziges Weltsystem aufweisen, liegen in manchen Erzählungen mehrere Weltsysteme vor, die zwei (oder mehr) disparate Teilmengen des diegetischen Raumes darstellen oder aber auf zwei (oder mehr) narrativen Ebenen lokalisiert sind und pluriregionale Welten genannt werden. 3. Zu differenzieren sind diejenigen Systeme, in denen die Handlung nach „,wechselnden Kriterien der Notwendigkeit und der Möglichkeit“ ${ }^{2}$ funktioniert, und solche, die über einen stabilen Kriterienkatalog verfügen. Die in Ersterem aufgebaute Instabilität kann entweder aufgelöst und in ein (vom Textende aus besehen) stabiles System überführt oder aber bis zum Schluss aufrecht erhalten werden. 4. Fiktive Welten können bzgl. ihrer physikalischen Gesetzmäßigkeiten und in logischer Hinsicht möglich oder unmöglich sein, insofern sie auf denselben Gesetzmäßigkeiten beruhen wie die extratextuelle Realität (= möglich) oder aber nicht (= unmöglich) und/oder sie ihrem Aufbau nach logisch kohärent (= möglich) oder aber paradoxal aufgebaut sind (= unmöglich).

Welten mit $\mathrm{Wb}$ sind heterogen, pluriregional oder instabil. ${ }^{3}$ Ein Analysevorhaben hat demzufolge zu bestimmen, welche Gesetzmäßigkeiten der Wahrscheinlichkeit, der Notwendigkeit und der Möglichkeit ein Weltsystem konstituieren - welches Wirklichkeitskonzept folglich zugrunde liegt - und welche innersystemische Divergenzen es gegebenenfalls offenbart, welche Transformationen es durchläuft oder welche Art von Transgressionen zwischen mehreren Weltsystemen stattfinden.

Die eigentlichen systemischen ,Brüche' in fiktionalen Texten entstehen jedoch nicht nur aufgrund der Existenz und der Gegenüberstellung von Wirklichkeitskonzepten, sondern werden durch Figurenhandlungen und/oder intersystemische Überlagerungen in der narrativen Vermittlung erzeugt. Folglich sind $\mathrm{Wb}$ auf unterschiedlichen narrativen Ebenen zu unterscheiden.

\footnotetext{
${ }^{2}$ Matías Martínez, Michael Scheffel: Einführung in die Erzähltheorie, 9. erw. u. aktual. Aufl., München 2012, S. 138.

${ }^{3}$ Logisch unmögliche Welten resultieren nicht aus der Divergenz zweier (oder mehrerer) Wirklichkeitskonzepte, sondern sind in sich paradoxal konzipiert. Daher stellen sie einen Sonderfall dar, der an dieser Stelle ausgeklammert wird.
} 
Auf der (sekundären) Ebene der Figurenhandlung werden Differenzen zwischen zwei Konzepten durch das Figurenwissen, ihre Bewegung und ihre Wahrnehmung verdeutlicht. Im Normalfall verfügen Figuren über einen mehr oder weniger explizierten Wissenstand bzgl. ihrer Umwelt, d.h. sie haben Kenntnis über Notwendigkeit, Wahrscheinlichkeit und Möglichkeit von Dingen und Geschehnissen. Sobald etwa ein Ereignis eintritt, das mit Hilfe des entsprechenden Wissens nicht erklärt werden kann, handelt es sich potentiell um einen Wb. Ein solcher Fall wird mit Höllriegels Die Films der Prinzessin Fantoche im Folgenden behandelt.

Die einfachste Form der Figurenbewegung ist diejenige, die die Figur im diegetischen Raum vollzieht: Eine Figur übertritt die Grenze zwischen zwei topografischen Räumen und mit ihr auch die Grenze zwischen zwei Systemen mit jeweils spezifischem Wirklichkeitskonzept. Im Zuge dieser Bewegung gilt für die Figur, das erlittene Ungleichgewicht auszugleichen und entweder dauerhaft in einem der beiden Räume zu verweilen oder aber beide Konzepte anzuerkennen - wobei letzteres die Notwendigkeit eines übergeordneten Wirklichkeitskonzeptes voraussetzt, das den gemeinsamen Bestand der auf den ersten Blick nicht zu vereinbarenden Konzepte zulässt. Carrolls Alice's Adventures in Wonderland (1865), worin die Protagonistin durch die Pforte zwischen einer realistischen Welt und einer märchenhaftphantastischen tritt und damit ihren Selbstfindungsprozess initiiert, behandelt eine solche Problematik.

Metaleptische Bewegungen von Figuren sind schwieriger zu fassen und in unterschiedliche Varianten aufgefächert. Die ontologische Metalepse meint die Versetzung einer Figur von einer narrativen Ebene in eine andere. ${ }^{4} \mathrm{~Wb}$ würden sich in diesem Fall durch die Gegenüberstellung zweier Wirklichkeitskonzepte auf unterschiedlichen narrativen Ebenen und dem daraus resultierenden Konflikt der Figur ergeben. Ein ,Bruch'vollzieht sich folglich durch die tatsächliche Bewegung eines Elementes des einen Systems (Figur) in das andere. ${ }^{5}$

Figurenwahrnehmung meint die perzeptiv-sinnliche Erfahrung der diegetischen Umwelt, darüber hinaus aber ebenso Wahrnehmungsprozesse, die beispielsweise durch bewusstseinserweiternde Katalysatoren herbeigeführt werden oder auf anderen ,übersinnlichen“ Wahrnehmungsvorgängen (wie Eingebungen, Einbildungen, Hellseherei sowie auf Träumen oder Trancezuständen) basieren. ${ }^{6}$ Entscheidend ist,

\footnotetext{
${ }^{4}$ Vgl. Gérard Genette: Die Erzählung, München 1998, S. 168.

${ }^{5}$ Verschiedene Varianten der metaleptischen Auseinandersetzung mit Wirklichkeit in Literatur und anderen Künsten behandelt ausführlich Sonja Klimak: Paradoxes Erzählen. Die Metalepse in der phantastischen Literatur, Paderborn 2010, insb. S. 247-279.

${ }^{6}$ Angesprochen sind damit rhetorische Metalepsen, bei der die Erzählinstanz (bspw. durch die Wahrnehmungssphäre einer Figur) eine andere diegetische Ebene andeutet: „Rhetorical metalepsis opens a small window that allows a quick glance across levels, but the window closes after a few sentences, and the operation ends up reasserting the existence of boundaries." (Marie-Laure Ryan: Avatars of Story, Minneapolis, MN, 2006, S. 207). Orth spricht in diesem Zusammenhang von Imaginationen, die ,typische Formen der hierarchisierten Pluralität“ hervorrufen (Orth: Narrative Wirklichkeiten, S. 159).
} 
welche Relevanz diese Wahrnehmungsformen für die Figur und die erzählte Welt haben. Wird über die Wahrnehmung einer Figur die Möglichkeit eines von dem durch die Erzählinstanz festgelegten Konzept abweichenden Systems eingeräumt und hat dies zudem Einfluss auf das Welt- und Wirklichkeitsverständnis der Figur oder der Erzählinstanz, so ist ebenfalls von Wb auszugehen. So offenbart Hofmanns The End (2008) im Nachhinein eine durch Filmexperimente forcierte Wahrnehmungsdeformation, die bis zu ihrer allmählichen Entdeckung durch den Protagonisten für phantastische Einbrüche in die realistisch gezeichnete Welt in Form von fiktiven Filmfiguren sorgt.

Allerdings fallen $\mathrm{Wb}$ und metaleptische Bewegungen von Figuren und figurale Wahrnehmungsstörungen nicht notwendigerweise zusammen. Versteht man unter ,Wirklichkeitskonzept ' die Gesamtmenge bestimmter für die Diegese geltenden oder zumindest von den dieser Menge zugeordneten Repräsentanten (Figuren) oder der diese Menge etablierenden Erzählinstanz für wahr gehaltenen Ordnungssätze ${ }^{7}$, so lassen sich schließlich alle in einem Text auftretenden Konzepte aufgrund ihrer explizit geäußerten oder implizit vorausgesetzten Parameter bestimmen und die Grenze zwischen ihnen anhand von Divergenzen benennen. Diese Grenze kann zwischen verschiedenen diegetisch-ontologischen oder narrativen Ebenen verlaufen, sie kann aber ebenso auf einer einzigen Ebene vorhanden sein. Die Erfassung dieser Grenze ist deshalb von Wichtigkeit für die Analyse, da an ihr punktuelle oder globale Brüche stattfinden, indem nämlich entweder ein Element der einen Wirklichkeitssphäre einmal oder mehrfach über die Grenze hinweg in eine andere versetzt wird oder in der Erzählerrede einmalige oder mehrfache Überlagerungen divergenter Wirklichkeitskonzepte vorliegen. ${ }^{8}$ Während Texte mit pluriregionalen Welten unterschiedliche Wirklichkeitssysteme ihrerseits eindeutig lokalisieren (Alice's Adventures in Wonderland), können Fälle solcher Überlagerungen in heterogenen und instabilen Welten auftreten: im einen Fall gelten - parallel und unveränderlich - Ordnungssätze disparater Konzepte (wie in Kafkas Die Verwandlung [1915]), im anderen changieren die geltenden Bedingungen (wie in Conan Doyles The Hound of the Baskervilles [1901/02]). In letzterem Fall gilt es dann zu überprüfen, an welcher Textstelle welche Parameter des einen Systems durch andere substituiert werden; in Ersterem, wie Figuren und Erzählinstanz mit den gegebenen Bedingungen umgehen: wird die

\footnotetext{
${ }^{7}$ Nach der Abwendung von einer Wirklichkeitsordnung a priori entpuppt sich Wirklichkeit mithin als Gesamtheit mehrerer, in einem bestimmten Zeitraum und einer jeweiligen Gesellschaft aufgestellten Annahmen über das, was als wirklich/der Wirklichkeit entsprechend verstanden wird. Vgl. Nelson Goodman: Ways of Worldmaking, Indianapolis 1978, S. 20.

${ }^{8}$ Lassen sich diese Überlagerungen auf die Intention einer Erzählinstanz zurückführen, so kann man mit Orth von ,absichtliche[n] Täuschungen“ oder aber von „Manipulationen der narrativen Wirklichkeit" sprechen (Orth: Narrative Wirklichkeiten, S. 184). Neben Formen dieser durch die Erzählinstanz hervorgerufenen Pluralisierungen existieren nach Orth ebenfalls Fälle von ,gleichberechtigten Pluralitäten“, aufgrund von 1. der Aufspaltung der narrativen Wirklichkeit, 2. einer paradoxalen narrativen Faktenlage, 3. der Präsentation sich widersprechender (Binnen-)Erzählungen ein und desselben Vorgangs (ebd., S. 197).
} 
Heterogenität der Diegese als solche unhinterfragt angenommen und sie dadurch potentiell getilgt oder stellt sie ein Problem dar?

Die Analyse der Ästhetik von Wb ist anschlussfähig an extratextuelle Wissensmengen und Diskurse der Frühen Moderne und Postmoderne, durch die oder in denen die Revision von Wirklichkeitsauffassungen offensichtlich virulent wird. Da Literatur - neben vielen anderen Produkten einer jeweiligen Kultur - als Speicher kulturellen Wissens $(\mathrm{kW})$ fungiert, rekurrieren auch Texte mit $\mathrm{Wb}$ auf bestimmte Diskurse im kulturellen Denksystem einer Epoche. ${ }^{9}$ Im Fall einer Auseinandersetzung mit $\mathrm{Wb}$ gilt es, die im $\mathrm{kW}$ propositional angelegte Problematisierung der jeweiligen Realitätsklassifikation - also dem, „was [...] für ,Realität ' gehalten wird“"10 - zu rekonstruieren und im Abgleich mit der Textanalyse zu berücksichtigen.

\section{Die Films der Prinzessin Fantoche: Kollision zwischen Illusion und Realität in der Frühen Moderne}

Höllriegels Die Films der Prinzessin Fantoche (1913) ${ }^{11}$ kann als ,Quasi'Kriminalroman gelesen werden. Er erzählt die Geschichte einer polizeilichen Jagd auf die Filmschauspielerin Prinzessin Fantoche, die einzelne Geschehnisse während dieser Jagd filmt und von der behördlichen Zensurstelle geprüft in die Kinos bringt - zunächst in die Lichtspielhäuser des Handlungsortes Genua, später in Kinos der ganzen Welt. Als ,Quasi'-Kriminalroman fungiert der Text deshalb, da er das Kriminalschema lediglich dazu nutzt, andere als solche in Kriminalerzählungen üblicherweise verhandelten Sachverhalte zu problematisieren: u.a. die Divergenz zwischen filmischer und literarischer Wirklichkeitskonzeption. Damit greift er einen für die 1910er Jahre zeittypischen Diskussionsgegenstand auf und ist insofern vielmehr Film- und Zeitroman. ${ }^{12}$

\footnotetext{
${ }^{9} \mathrm{~kW}$ wird als „Gesamtmenge aller der von den Mitgliedern einer Kultur, das heißt in einer bestimmten Zeitphase T [...] und in einem bestimmten Raum R [...], für wahr gehaltenen Propositionen“" definiert. Zugänglich wird es über sprachliche und nicht-sprachliche Wissensquellen und ist analytisch zu rekonstruieren. (Michael Titzmann: Propositionale Analyse - kulturelles Wissen - Interpretation, in: Medien und Kommunikation. Eine interdisziplinäre Einführung, hrsg. von Hans Krah, Michael Titzmann, 2., überarb. Aufl. Passau 2011, S. 83-108, hier S. 90).

${ }^{10}$ Ebd., S. 93.

${ }^{11}$ Arnold Höllriegel ist das Pseudonym des Schriftstellers Richard A. Bermann.

${ }^{12}$ Vgl. Michael Grisko: Simulationen, Sensationen, Serialitäten. Richard A. Bermann alias Arnold Höllriegel entdeckt den Film für die Literatur und begründet ein neues Genre, in: Arnold Höllriegel: Die Films der Prinzessin Fantoche, hrsg. von Michael Grisko, Berlin 2003, S. 138-156, hier S. 139 u. 151 u. Andrea Haller: Shadows in the Glasshouse. Film Novels in Imperial Germany, 1913-1917, in: Film History 20/2 (2008), S. 164-180, hier S. 166.
} 
Gleichwohl - wie sich am Ende herausstellt - von Prinzessin Fantoche kein Verbrechen begangen wird, sind die Grundzüge eines Krimis durchaus vorhanden. ${ }^{13}$ Als Auslöser der Geschichte dienen zwei in der fiktiven Vergangenheit liegende Umstände: die finanzielle Notlage des Filmstudios OCI einerseits und der Beginn der über den Zeitpunkt des Erzähleinsatzes hinaus anhaltenden Liebesbeziehung zwischen Marie-Madelaine Fanny d'Estourbiac - welche zunächst als Marie Dupont auftritt und sich im weiteren Verlauf als Prinzessin Fantoche entpuppt - und Eugenio Testaccia, dem Sohn des Genueser Polizeichefs, andererseits. Ebenfalls in der fiktiven Vergangenheit positioniert, jedoch im Anfangskapitel rekapituliert, spielt sich das vermeintliche Verbrechen ab: Der wohlhabende Geschäftsmann Ippoliti plant als Überraschung für die Gäste seiner Geburtstagsfeier einen fingierten Überfall als Film vorzuführen. Prinzessin Fantoche und ihre Helfer treffen bei ihm ein, um die Rahmenbedingungen zu besprechen und den Film zu drehen. Dabei wird offenbar, dass es sich bei dem Filmteam um tatsächliche Einbrecher handelt: das von Ippoliti fingierte Verbrechen wird zu einem realen. Die Auflösung des Falles erfolgt im weiteren Verlauf allerdings nicht durch die Ermittler, sondern dadurch, dass sich Prinzessin Fantoche der Polizei stellt. Ihre Filme entpuppen sich in einem aufklärenden Gespräch zwischen Fantoche und Testaccia als marketingstrategischer PR-Gag der Genueser Filmproduktionsfirma OCI: Ippoliti hatte als Chef dieser Firma selbst in Auftrag gegeben, den Umschwung von einem fingierten in einen tatsächlichen Überfall zu inszenieren. Fantoches Motivation wiederum, ihre Liebesbeziehung zu Eugenio zu legitimieren, wird dem Commendatore erst offengelegt, als jener dieser Legitimation nicht mehr entgegenwirken kann.

Maßgeblich für die Bedeutungskonstitution des Textes ist neben der schematisch-trivialen Folie des Kriminalfalles die Problematisierung von Wirklichkeit und die Auseinandersetzung mit bestimmten Formen der narrativen Gestaltung. Analytische Ansatzpunkte bilden dabei erstens das im Roman exemplifizierte Umspielen der Grenze zwischen filmischer Illusion und fiktionsinterner Realität und die daraus resultierende Verunsicherung der Figuren hinsichtlich ihres Verhaltens und hinsichtlich der Gesetzmäßigkeiten der Diegese sowie zweitens die Gegenüberstellung von literarischem und filmischem Erzählen. Die latente Gefahr einer Übertragung realer Ereignisse in einen Film auf der einen Seite und ihr Nutzen auf der anderen sowie Effekte des Filmens auf die erzählten Geschehnisse laufen im ersten Punkt zusammen und betreffen die sekundäre Ebene der Figuren. Das im zweiten Punkt genannte Problem wird auf der primären Ebene des Erzählens verhandelt und ist ausschlaggebend für die Gestaltung von Histoire und Discours.

\footnotetext{
${ }^{13}$ Diese beinhalten eine zeitlich vor dem Erzähleinsatz positionierte Vorgeschichte, die die Figurenhandlung motiviert und die im Laufe der Geschichte rekonstruiert wird; einen Fall in Form eines Verbrechens oder Anzeichen eines solchen, der im Zuge der Detektion aufgeklärt wird; und die ,Sühnung' des Straftäters. Vgl. Edgar Marsch: Die Kriminalerzählung. Theorie - Geschichte - Analyse, München 1972, S. 17.
} 
Die Films der Prinzessin Fantoche etabliert zwei Realitätskonzepte (K1= , Realität'/K2= ,Film') und damit korrelierte Realitätssphären (S1/S2). Beide Konzepte/Sphären wie auch die Grenze zwischen ihnen werden über das Figurenwissen installiert. D.h. der Text führt zwei Figurengruppen vor, diejenige Gruppe, die über ein Wissen bzgl. K1/2 und S1/2 verfügt $\left(\mathrm{F}_{1,2}\right)$, und diejenige Gruppe, die hinsichtlich $\mathrm{K} 2$ und $\mathrm{S} 2$ ein Wissensdefizit aufweist $\left(\mathrm{F}_{1}\right)$. Wissenslücken der ,ahnungslosen Verfolger “'14 offenbaren sich ganz deutlich an mehreren Stellen im Text. Eine - wie sich später herausstellt - lediglich vermeintliche Lücke findet sich bei Ippoliti: „Jetzt begriff er [Ippoliti] die schreckliche Wahrheit.“15 Diese Wahrheit vermag er indes nicht zu ertragen und fällt in Ohnmacht. Vor allem sind es die Ordnungshüter, die K2 bis zum Schluss nicht zu durchdringen imstande sind, obwohl sie selbst vor allem wiederholt und insbesondere Objekte von S2 sind (indem sie gefilmt werden): ${ }^{16}$ „Ich sehe, ich habe eine furchtbare Dummheit gemacht! Aber wer konnte ahnen..." “17, äußert sich Cavaliere Depretis. Und ebenso gibt der Innenminister Ghezzi nach einer Verfolgung der Fantoche zu: „Wir hätten uns denken können, daß sie gescheiter sein würde als wir. “18 Aber auch der als ,leicht zu begeisternde[r] Phantast $^{\text {“19 }}$ eingeführte Eugenio Testaccia spricht, nachdem seine Geliebte sich ihm gegenüber als Prinzessin Fantoche zu erkennen gibt, von einer „verrückte[n] Halluzination. “20

Die entscheidende Divergenz beider Figurengruppen besteht darin, dass das Wissen von $F_{1,2}$ in existentieller Hinsicht vorteilhaft erscheint. $F_{1,2}$ dominiert $F_{1}$, darüber hinaus sind Teilnehmer von $F_{1}$ potentiell zum Scheitern verurteilt. Dieses Scheitern hat eine qualitative Komponente und verläuft graduell. Qualitativ äußert es sich in wirtschaftlicher, beruflicher oder persönlicher Hinsicht. Freilich in Form einer filmischen Inszenierung - bis zuletzt aber aus Sicht von $\mathrm{F}_{-2}$ glaubhaft -, verliert Ippoliti beim Raub durch die Prinzessin Fantoche nahezu sein gesamtes Vermögen (= wirtschaftliches Scheitern). Trotz aller Bemühungen kommen Depretis, Testaccia und Ghezzi Prinzessin Fantoche bis zum Schluss nicht auf die Spur und werden darüber hinaus in allen Belangen vorgeführt (= berufliches Versagen). Seine Unkenntnis von K2, vor allem aber sein unbeirrtes Festhalten an den Prinzipien von $\mathrm{K} 1$, lassen Testaccia schließlich insofern persönlich scheitern, als er am Ende seinen Sohn in zweierlei Hinsicht verliert: er versagt in der Weitergabe seines Berufstands

\footnotetext{
${ }^{14}$ Höllriegel: Fantoche, S. 52.

${ }^{15}$ Ebd., S. 20.

${ }^{16}$ Dieser Umstand ist aus Sicht von $F_{1}$ potentiell zu sanktionieren. So äußert sich Commendatore Testaccia seinem tollpatschigen Angestellten Depretis gegenüber: „Sind Sie ein Komiker oder ein königlicher Polizeibeamter? Als Komiker haben Sie Erfolge. Als Beamter sind Sie unfähig. Glauben Sie, der Staat zahlt Ihnen Ihr Gehalt, damit sie [sic!] als humoristischer Kinodarsteller Sensation machen?" (Höllriegel: Fantoche, S. 56).

${ }^{17}$ Höllriegel: Fantoche, S. 31 (Herv. von mir, S.B.).

${ }^{18}$ Ebd., S. 102 (Herv. von mir, S.B.).

${ }^{19}$ Ebd., S. 44.

${ }^{20}$ Ebd., S. 73.
} 
an seinen Sohn und muss das Liebespaar ohne eigene Entscheidungsmacht ziehen lassen. Graduelle Unterschiede lassen sich anhand des Ausmaßes des Scheiterns erkennen: Während das temporär berufliche Versagen für Depretis und Ghezzi wohl keine längerfristigen Folgen nach sich zieht, ist Testaccias Verlust seines Sohnes (und damit der familiären Zukunft) als sehr viel schwerwiegender einzuschätzen. Festzuhalten ist, dass Figuren im Spannungsfeld zwischen beiden Sphären angesiedelt sind und nur diejenigen triumphieren, die die Existenz beider Konzepte nicht nur akzeptieren, sondern sie (mehr [Fantoche] oder weniger [Eugenio]) durchdringen. ,Triumphieren' korreliert hier die Aussicht auf eine positive Zukunft und persönliches Glück als realisierte Option neben anderen möglichen.

Die Problematik der Histoire lässt sich ebenfalls auf der Discours-Ebene wiederfinden. Die Ebene der narrativen Vermittlung enthält die Selektion und Serialisierung von Attraktionsmomenten (bspw. die Verfolgungsjagd, Kap. 6), die Darstellung von Bewegung und die Reflexion medialer Repräsentationen. Doch aller zu berücksichtigenden filmischen Inhalte und Elemente zum Trotz ist der literarische Impetus unleugbar der dominierende. Denn gegenüber anderen literarischen Texten filmischen Erzählens - wie etwa die kurzen Kinostücke in Pinthus' Kinobuch (1913) -, sind filmische Komponenten, gleichwohl sie die histoire bestimmen, nur partiell in den discours integriert. Dahingegen überwiegen dezidiert sprachliche Darstellungen der Erzählinstanz (so das erste rekapitulierende Kapitel) und der Figuren (Dialoge) sowie das Mittel der Introspektion (insbesondere mit Blick auf Vater und Sohn Testaccia). Beides läuft augenscheinlich in der ,neuen' Gattung des Filmromans zusammen. Am freien Umgang mit Elementen des Kriminalromans lässt sich an ihm ein Findungsprozess literarischen Erzählens ablesen, der wiederum am ,neuen` Medium Film orientiert ist und von den Möglichkeiten filmischen Erzählens profitiert. Mit anderen Worten liegt ein literarisches Plädoyer für das filmische Erzählen als Form literarischen Erzählens vor. ${ }^{21}$

Auf einem höheren Abstraktionsniveau verhandelt Die Films der Prinzessin Fantoche die ebenfalls in der Kino-Debatte diskutierte Frage nach der Wirklichkeitsadäquatheit von Künsten, genauer: ob nicht die Moderne etwa in der Kunstform Film sehr viel deutlicher und angemessener zum Ausdruck gelange als in der tradierten Kunst Literatur. Eine Grundlage dieses Wirklichkeitsdiskurses stellt die Großstadt dar, die das Widersprüchliche vereint, Simultaneität und Diskontinuität „unzusammenhängender Bewegungen“222 forciert und beim Einzelnen schockartige Eindrücke hervorruft. Das großstädtische Ensemble gilt als Symbol der modernen alltäglichen Wirklichkeit, denn mit ihm verbunden ist die Symptomatik des literarischen Umgangs mit Realität: das gefühlte Unvermögen, Wirklichkeit sprachlich zu erfassen. Während das Credo des 19. Jahrhundert mit der ,Zuwendung zur Gesamt-

\footnotetext{
${ }^{21}$ Zur Ausformung filmischen Erzählens als Form literarischen Erzählens im Kontext der Frühen Moderne vgl. die unlängst erschienene Publikation meiner Dissertation Filmisches Erzählen. Typologie und Geschichte, Berlin/Boston 2014, S. 188-219.

${ }^{22}$ Joachim Paech: Literatur und Film, 2., überarb. Aufl., Stuttgart/Weimar 1997, S. 125.
} 
wirklichkeit' etikettiert werden könnte, zeichnet sich mit Hofmannsthals Brief des Lord Chandos (1901) - und damit symptomatisch für die Frühe Moderne - eine entscheidende Wende des Verhältnisses der Literatur zur Wirklichkeit ab. Denn

[...] jetzt wird die Realität als solche fragwürdig, die Dinge entgleiten dem Zugriff des Subjekts, verselbständigen sich, gewinnen Autonomie [...]. [D]ie Fremdheit einer nicht mehr faßbaren Welt steht am Anfang. Die Ordnung zerbricht in Teile, die Teile lösen sich in Teilchen auf, die Begriffe versagen. $^{23}$

Zwar besteht auch um die Jahrhundertwende nach wie vor die Ausrichtung auf ,die' außerliterarische Wirklichkeit, aber ,wie sollte jemand, der an der Ordnung der Welt zweifelt, noch an den Kosmos der Syntax und die heiligen Regeln der Grammatik glauben?‘ ${ }^{24}$ Die Großstadt repräsentiert den Umbruch in sozialer, kultureller, technologischer und ökonomischer Hinsicht. In ästhetischer Hinsicht fungiert die Sprachskepsis als Katalysator für verschiedene literarische Stiltendenzen und ist Ausgangspunkt für die Ausformung einer neuen, filmbasierten Literaturpoetik, wie sie explizit Döblin (Kinostil [1913]) und Pinthus (Das Kinobuch) und zumindest implizit Höllriegel ins Leben gerufen haben.

Auch die Kino-Debatte greift die „Krise des Wortes“棌 wird, der Sprache hafte „etwas merkwürdig Undifferenziertes“26 an. Zugleich hebt man die Möglichkeiten des Films hervor, der dem Großstadt-Dispositiv mit seiner gestalteten Visualität zu entsprechen vermöge und der darüber hinaus einen neuen fiktionalen Ort des Phantastischen böte. ${ }^{27}$ Der scheinbaren Ablösung des Romans durch den Film, seinem vermeintlichen gesellschaftlichen Abstieg sowie seinem ästhetischen Geltungsverlust wirken u.a. Döblin, Pinthus, Höllriegel dadurch entgegen, dass sie auf verschiedene Weise filmische Verfahren und inhaltliche Konzepte des Films zu integrieren versuchen. Döblins Kinostil bspw. meint eine bestimmte

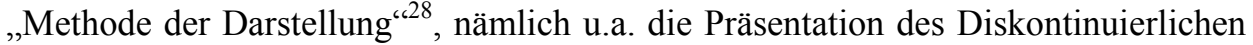
und die Rücknahme der Erzählinstanz zur distanzlosen Wiedergabe des Dargestellten, Pinthus konzentriert sich in der Skizze seines Kinostückes auf die Aspekte ,Milieu', ,Bewegung' und ,Trick ${ }^{\text {(29 }}$, während Höllriegel in seinem ästhetischen Konglomerat aus Kriminal-, Liebes- und Actionroman und der Verzahnung literarischer und filmischer Erzählelemente mittels $\mathrm{Wb}$ einen praktischen Weg aufzeigt, eine Narration des Übergangs zu schaffen.

\footnotetext{
${ }^{23}$ Walter Jens: Moderne Literatur - Moderne Wirklichkeit, Metzingen/Württemberg 1958, S. 12.

${ }^{24}$ Jens: Moderne Literatur, S. $12 \mathrm{f}$.

${ }^{25}$ Anton Kaes: Die Kino-Debatte. Texte zum Verhältnis von Literatur und Film. 1909-1929, München/Tübingen 1978, S. 17.

${ }^{26}$ Egon Friedell: Prolog vor dem Film, in: Kaes: Kino-Debatte, S. 42-47, hier S. 45.

${ }^{27}$ Vgl. Kaes: Kino-Debatte, S. 7.

${ }^{28}$ Vgl. Alfred Döblin: An die Romanautoren und ihre Kritiker. Berliner Programm, in: ders.: Aufsätze zur Literatur, Olten [u.a.] 1963, S. 15-19, hier S. 17.

${ }^{29}$ Vgl. Kurt Pinthus: Einleitung: Das Kinostück, in: Das Kinobuch, hrsg. von Kurt Pinthus, Zürich 1963, S. 19-33, hier S. 25ff.
} 


\section{Replay: Die postmoderne Diffusion von ,Realitäten'}

Anders als Die Films der Prinzessin Fantoche realisiert Steins Replay (2012) $\mathrm{Wb}$ in Form von unauflösbar ineinander verschlungenen Realitätskonzepten im narrativen Akt. Die erzählte Geschichte weist drei wesentliche Dimensionen auf. Zum ersten handelt es sich um die Geschichte des Erzählers selbst - Ed Rosen -, der bereits vor dem Erzähleinsatz drei wesentliche physische und psychische Transformationsschritte durchlaufen hat, nämlich 1. die Entdeckung des eigenen Körpers und die damit verbundene Aufwertung von Körperlichkeit aufgrund der beginnenden bewussten Wahrnehmung körperlicher Merkmale und Bedürfnisse (T1); 2. die Implantation des sog. UniComs - eine im weitesten Sinne als Kommunikations- und Aufnahmegerät zu begreifende technische Vorrichtung - samt dem für die Operation notwendigen und durch Ernährungsumstellung und Fitnesstraining forcierten ,Aufbau' des Körpers (T2); und schließlich 3. den Aufstieg zum Minister der erzählten Welt, der mit sozialer Macht, zugleich aber auch mit erneuter sozialer Isolation korreliert ist (T3).

Mit der Geschichte des Erzählerprotagonisten verbunden ist zum zweiten eine Liebesgeschichte, zunächst zwischen Ed Rosen und Katelyn, die er als Arbeitskollegin kennenlernt und die im weiteren Verlauf als seine persönliche Fitnesstrainerin eingesetzt wird, später dann in Form einer Dreiecksbeziehung zwischen Ed, Katelyn und Lian, einer chinesischen Masseurin. Die Entwicklung dieser Beziehung basiert vor allem auf der Erfahrung von Sexualität und der Rezeption sogenannter Replays (das sind mittels UniCom erzeugte und konservierte Medienformate zwecks retrospektiver und multisensueller [Wieder-]Erfahrbarkeit von erlebten und vergangenen Wahrnehmungsmomenten). Die Beziehung ist aber außerdem geprägt durch ein Spiel mit Vertrauen, welches in schärfster Form durch Eds Vergewaltigungsversuch an Lian zutage tritt und ausschlaggebend für die Auflösung der Liebesbeziehung sowie für Katelyns und Lians Flucht ist.

Drittens schließt sich an die Entwicklung des UniComs ein Wandlungsprozess der dargestellten Welt an von einer realistisch gezeichneten, der Lebenswirklichkeit des frühen 21. Jahrhunderts angelehnten Welt hin zu einer futuristischen, die durch technologisch bedingte Gleichschaltung des Menschen und die der Gesellschaft aufoktroyierte monopolistische Hegemonialstellung des Konzerns um Ed Rosen geprägt ist. Maßgebend für diese Entwicklung ist die Erfindung und sukzessive technologische Optimierung des UniComs, das als bahnbrechende Medientechnologie Wahrnehmung(-sfähigkeit) und Kommunikation der menschlichen Gesellschaft zunächst revolutioniert und in der Folge manipuliert. Medienfortschritt wird hier demnach mit sozialer Entwicklung korreliert und entgegen Eds sozialem Aufstieg das negative Bild einer anti-utopischen Welt gezeichnet.

Die Auffassung über das, was Wirklichkeit ist, und die im Erzählprozess verhaftete Transgression von Wirklichkeitsgrenzen sind als wesentliche Elemente der Be- 
deutungskonstitution des Textes aufzufassen. Im Zentrum steht dabei Ed Rosen, das erzählende Subjekt. Nicht nur, dass er als Erzählinstanz die von ihm erzählte Welt im narrativen Akt überhaupt erst hervorbringt, ausschlaggebend ist darüber hinaus die Labilität des gesamten fiktiven Realitätsgefüges. Deutlich wird dies einerseits anhand des leitmotivisch wiederkehrenden Pan - eine Gestalt, die der Erzähler aus del Torros Film El laberinto del fauno (E/MEX/USA 2006) adaptiert und als ,Marker' für ,erinnerte Realität' in seine Replays einarbeitet (dies jedoch ebenso mancherorts bewusst unterlässt) - und anhand der am Ende der nur wenige Stunden eines Morgens umfassenden Erzählung realisierten Rückbindung an den Erzählbeginn und die direkte Wiederholung der Eingangspassage. Letzteres legt offen: das Erzählersubjekt befindet sich in einer Art Zeitschleife und einer manipulativ deformierten Wirklichkeit und überträgt seinen Zustand auf die den geschilderten Ereignissen zugrundeliegende bzw. ,ursprüngliche` Realität.

Dies wiederum hat zweierlei zur Folge: Erstens ist dem Erzähler nicht zu trauen, er muss als unzuverlässiger Erzähler angesehen werden. Zweitens setzt der Text zwei Wirklichkeiten in ein Spannungsverhältnis, wobei die eine die zeitlich vor T2 gesetzte (ursprüngliche) Wirklichkeit (W1) und die andere die sich nach T2 konstituierende, deformierte Wirklichkeit (W2) darstellt. Angesichts des Erzählzeitpunkts (nach T3) muss betont werden, dass W2 die gesamte Erzählung überlagert. Zu folgern ist aber weiterhin, dass die Implikation der, Wirklichkeitsdeformation' über T3 hinaus Bestand hat: Dass (nur) einige Replays mit dem Marker , unwirklich` (Pan und Pans Insignien) versehen sind, bedeutet in der logischen Fortführung: alle erzählten Geschehnisse mit Marker sind ,unwirkliche' (= nicht-aktuelle und technisch bearbeitete) Geschehnisse. W1 ist in Anbetracht dessen u.a. deshalb greifbar, da der Erzähler Referenzpunkte zur extratextuellen Realität setzt (durch Eigennamen wie Assange, Zuckerberg, Jobs oder durch Bezüge zu technologischen Standards des frühen 21. Jahrhunderts, wie etwa Smartphones). Da aber Pan bereits von Beginn an und in der Folge über die gesamte Erzählung verteilt auftaucht und der Erzählstandort nicht wechselt, ist davon auszugehen, dass die Narration insgesamt die Summe mehrerer Replays darstellt. Zwar wird vom Erzähler wiederholt das Bedürfnis nach Erinnerung an den Abend, der vor dem Erzähleinsatz liegt, geäußert. Aber gleichwohl die vergangenen Ereignisse im Verlauf der Erzählung rekonstruiert werden, muss man zu dem Schluss kommen, dass der Erzähler seine Erinnerungsfähigkeit gänzlich eingebüßt hat und er im Akt des Erzählens nicht auf die eigenen Erinnerungen, sondern auf technisch-aufbereitete (und in dieser Hinsicht - so legt der Text nahe - grundsätzlich fragwürdige) Replays zugreift.

Der Erzähler erzeugt - so lässt sich weiter folgern - erstens eine Narration, deren diegetische Realität unterhöhlt ist. W1 ist gleichermaßen präsent und absent bzw. durch ein deformiertes Substitut neubelegt. Zweitens steht das Subjekt im Spannungsfeld zwischen Linearität und Zirkularität. Erzählt wird der individuelle Werdegang eines sozialen Aufstiegs, eingebettet ist diese (lineare) Entwicklung 
allerdings in die Schleife des zirkulär auftretenden narrativen Neuanfangs. ${ }^{30}$ Vergangenheit und Zukunft werden im Rahmen dieses Erzählmodells potentiell ausgehebelt, ihre Wertigkeit dadurch aber angehoben: dass Ed Rosen als ,Semiotiker" eingeführt wird, ist deshalb relevant, da seine Fähigkeit, Zeichen zu deuten, zum Zeitpunkt nach T3 offensichtlich mehr als beeinträchtigt ist. Was die Zukunft betrifft, so ist die Realisierung eines persönlichen Glückes aufgrund des Liebesverlustes wohl unmöglich. Weiterhin scheint die technologische Revolution ,ihre eigenen Kinder zu fressen‘. Denn am Schluss der Erzählung nähern sich Eds ,gläsernem Palast ${ }^{`}$ Ordnungshüter, um Ed zu verhaften. Drittens markieren Wb den Bezugsverlust des Subjekts zur Realität. Dieses Scheitern äußert sich selbstverschuldet und existentiell. Graduell wie auch qualitativ steuert die Figur in das Extrem: Liebe und Lust münden in Gewalt, Erfolg gerät zu einem Übermaß an Arbeitsaufwand, das Speichern der Vergangenheit zerstört Gegenwart und Zukunft, technologisch ermöglichte Kommunikationsoffenheit bewirkt soziale Isolation und Überwachung usw.

Replay verhandelt damit eine Reihe postmoderner Diskurse. Trägt man bspw. Hassans Merkmalskatalog zur Postmoderne an den Text heran, so werden Technologismus, Dehumanisierung, Erotizismus, Fragmentierung, Offenheit, Heterogenität, Hybridisierung und Konstruktivismus als Themen oder strukturgebende Elemente evident. ${ }^{31}$ Zentral ist in diesem Zusammenhang Hassans Begriff indeterminacy ${ }^{32}$ und das damit verbundene Merkmal der narrativen Selbstreflexivität (Metafiktion). Der Text konstituiert Bedeutung nämlich auf zwei Ebenen: Auf der ersten Ebene wird die ambivalente Geschichte des sozialen Aufstiegs des Protagonisten einerseits und sein ethischer Abstieg sowie sein Bezugsverlust zur Realität andererseits erzählt. Zum anderen manifestiert sich dieses Scheitern auf der Ebene des Erzählens, insofern die deformierte Wahrnehmung des Erzählersubjekts eine Dekonstruktion des Realitätsgefüges zur Folge hat. Mit anderen Worten: Das zirkuläre Erzählen ist Resultat des Erzählten, das es gleichsam dekonstruiert.

Ebenfalls augenfällig ist die Auseinandersetzung mit Baudrillards ,Theorie der Simulation“ und die „Substituierung des Realen durch Zeichen des Realen“333, die

\footnotetext{
${ }^{30}$ Diesem Aspekt postmodernen Erzählens bin ich ebenfalls in Nolans Memento (USA 2000) nachgegangen (vgl. Stephan Brössel: Zeit und Film. Zeitkreise in Christopher Nolans „Memento “, in: Zeit(en) erzählen. Ansätze - Aspekte - Analysen, hrsg. von Lukas Werner, Antonius Weixler [in Vorbereitung]).

${ }^{31}$ Vgl. u.a. Ihab Hassan: POSTmodernISM. A Paracritical Bibliography (1971), in: Postmodernism in American Literature. A Critical Anthology, hrsg. von Manfred Pütz, Peter Freese, Darmstadt 1984, S. 82-95.

${ }^{32}$ Ihab Hassan: Culture, Inderterminacy, and Immanence. Margins of the (Postmodern) Age, in: ders.: The Postmodern Turn. Essays in Postmodern Theory and Culture, Ohio State University 1978, S. 46-83, hier S. 73.

${ }^{33}$ Jean Baudrillard: Die Präzision der Simulakra, in: ders.: Agonie des Realen, Berlin 1978, S. 7-70, hier S. 9.
} 
eine Hyperrealität hervorruft, worin sich wiederum das Reale verflüchtigt ${ }^{34}$ : Es bleibt mit Blick auf die gesamte Erzählung schlicht nicht entscheidbar, ob der Erzähler seine eigenen oder medial konservierte und damit potentiell manipulierte Replays präsentiert. Dadurch wird in Frage gestellt, was Realität überhaupt sein soll - ist sie nicht immer Produkt eines Konstruktionsprozesses? Man kann mit Baudrillard sogar noch einen Schritt weitergehen und unterstellen, dass nicht nur die Simulation der Realität vorliegt, sondern der Text den Mechanismus der Dissuasion vorführt. ${ }^{35}$ Denn offen bleibt, ob Replay eine scheinbare Realität in einer Hyperrealität simuliert oder ob nicht etwa diese Hyperrealität sogar absolut gesetzt wird, indem sie vielmehr die „Absenz der Realität vertuscht ${ }^{\star 36}$. Folglich sind $\mathrm{Wb}$ entscheidende Erzählmittel zur Verhandlung der eigentlichen Problematik: Es geht vornehmlich nicht um die bloße Gegenüberstellung zweier Realitäten (der gegenwärtigen Wirklichkeitssphäre und der Sphäre eines synästhetischen Medienprodukts), sondern um das Scheitern der Figur im postmodernen Erzähltext. Mit den mit Hilfe von narrativen $\mathrm{Wb}$ verhandelten Diskursen führt Replay daher vor Augen, dass Modelle der Postmoderne noch immer präsent sind. Gleichsam stellt die Sehnsucht des Erzählers nach einer geordneten, widerspruchslosen Erzählung mit deutbaren Geschehnissen, die er selbst nicht zu schaffen vermag, das Signum eines narrativen Übergangs dar.

Mit Die Films der Prinzessin Fantoche und Replay lagen zwei Texte der Frühen Moderne und der Postmoderne vor, an denen die theoretischen Überlegungen zur narrativen Inszenierung von Wirklichkeitsbrüchen illustriert wurden. Die Analysen konnten offenlegen, dass beide Texte Wirklichkeitsbrüche in allen drei genannten Bereichen der Diegese, der narrativen Vermittlung und der Figurenhandlung realisieren und durch die Art und Weise ihrer narrativen Gestaltung Narrationen epochaler Übergange inszenieren. Während Höllriegels Text im medienhistorischen und sprachskeptizistischen Kontext der Frühen Moderne gelesen werden kann und die Grenze zwischen Filmphantastik und Realität auslotet, um eine neue literaturästhetische Poetik zu etablieren, hebt Steins Roman auf die Problematisierung postmoderner Phänomene $a b$ und umkreist die Frage nach der Konstitution des Subjekts im digitalen Zeitalter. Im einen Fall kann von einer progressiven Öffnung gegenüber neuen Erzählmustern zur Überwindung einer literarästhetischen Krise ausgegangen werden, im anderen vom semiotischen Zusammenhang zwischen Erzählen und aktueller Mediengeschichte sowie internationaler Kommunikationspolitik. Das Phänomen des ästhetischen Wirklichkeitsbruchs ist in beiden Fällen als Signal zu lesen und die Gegenüberstellung zweier Wirklichkeitskonzepte im Text als zeichenhaft für Prozesse außerhalb des Textes aufzufassen.

\footnotetext{
${ }^{34}$ Vgl. Jean Baudrillard: Der symbolische Tausch und der Tod, München 1982, S. 114.

${ }^{35}$ Zur Dissuasion in Abgrenzung zur Simulation vgl. Baudrillard: Die Präzision der Simulakra, S. 7-69.

${ }^{36}$ Christer Petersen: Der postmoderne Text. Rekonstruktion einer zeitgenössischen Ästhetik am Beispiel von Thomas Pynchon, Peter Greenaway und Paul Wühr, Kiel 2003, S. 216.
} 


\section{Literatur}

Baudrillard, Jean: Die Präzision der Simulakra, in: ders.: Agonie des Realen, übers. von Lothar Kurzawa und Volker Schaefer, Berlin 1978, S. 7-69.

Baudrillard, Jean: Der symbolische Tausch und der Tod, übers. von Gerd Bergfleth, München 1982.

Brössel, Stephan: Filmisches Erzählen. Typologie und Geschichte, Berlin/Boston 2014.

Brössel, Stephan: Zeit und Film. Zeitkreise in Christopher Nolans „Memento“, in: Zeit(en) erzählen. Ansätze - Aspekte - Analysen, hrsg. von Lukas Werner, Antonius Weixler [in Vorbereitung].

Carroll, Lewis: Alice's Adventures in Wonderland, Reprint, London [u.a.] 1964.

del Torro, Guillermo: El laberinto del fauno (E/MEX/USA 2006).

Doyle, Arthur Conan: The Hound of the Baskerville, Oxford 1994.

Döblin, Alfred: An die Romanautoren und ihre Kritiker. Berliner Programm, in: ders.: Aufsätze zur Literatur, Olten [u.a.] 1963, S. 15-19.

Friedell, Egon: Prolog vor dem Film, in: Kaes, Anton: Die Kino-Debatte. Texte zum Verhältnis von Literatur und Film. 1909-1929, München/Tübingen 1978, S. 42-47.

Genette, Gérard: Die Erzählung, übers. von Andreas Knopp, München 1998.

Goodman, Nelson: Ways of Worldmaking, Indianapolis 1978.

Grisko, Michael: Simulationen, Sensationen, Serialitäten. Richard A. Bermann alias Arnold Höllriegel entdeckt den Film für die Literatur und begründet ein neues Genre, in: Höllriegel, Arnold: Die Films der Prinzessin Fantoche, hrsg. von Michael Grisko, Berlin 2003, S. 138-156.

Haller, Andrea: Shadows in the Glasshouse. Film Novels in Imperial Germany, 1913-1917, in: Film History 20/2 (2008), S. 164-180.

Hassan, Ihab: Culture, Indeterminacy, and Immanence. Margins of the (Postmodern) Age, in: ders.: The Postmodern Turn. Essays in Postmodern Theory and Culture, Ohio State University 1978, S. 46-83.

Hassan, Ihab: POSTmodernISM. A Paracritical Bibliography, in: Postmodernism in American Literature. A Critical Anthology, hrsg. von Manfred Pütz, Peter Freese, Darmstadt 1984, S. 82-95.

Hofmann, Ulrich: The End, Berlin 2008.

Höllriegel, Arnold: Die Films der Prinzessin Fantoche, hrsg. von Michael Grisko. Berlin 2003.

Jens, Walter: Moderne Literatur - Moderne Wirklichkeit, Metzingen/Württemberg 1958.

Kafka, Franz: Die Verwandlung, in: ders.: Drucke zu Lebzeiten, hrsg. von Wolf Kittler, Hans-Gerd Koch u. Gerhard Neumann. Frankfurt a.M. 2002 (= Schriften, Tagebücher. Kritische Ausgabe).

Das Kinobuch, hrsg. von Kurt Pinthus, Zürich 1963.

Klimak, Sonja: Paradoxes Erzählen. Die Metalepse in der phantastischen Literatur, Paderborn 2010.

Marsch, Edgar: Die Kriminalerzählung. Theorie - Geschichte - Analyse, München 1972.

Martínez, Matías/Scheffel, Michael: Einführung in die Erzähltheorie, 9. erw. u. aktual. Aufl., München 2012.

Orth, Dominik: Narrative Wirklichkeiten. Eine Typologie pluraler Realitäten in Literatur und Film, Marburg 2013.

Paech, Joachim: Literatur und Film, 2., überarb. Aufl., Stuttgart/Weimar 1997.

Petersen, Christer: Der postmoderne Text. Rekonstruktion einer zeitgenössischen Ästhetik am Beispiel von Thomas Pynchon, Peter Greenaway und Paul Wühr, Kiel 2003.

Ryan, Marie-Laure: Avatars of Story, Minneapolis, MN, 2006.

Stein, Benjamin: Replay, München 2012.

Titzmann, Michael: Propositionale Analyse - kulturelles Wissen - Interpretation, in: Medien und Kommunikation. Eine interdisziplinäre Einführung, hrsg. von Hans Krah, Michael Titzmann, 2., überarb. Aufl., Passau 2011, S. 83-108. 